\title{
Automated Line-Based Sequential Sampling and Modelling Algorithm for EMC Near-Field Scanning
}

\author{
Prashant Singh, Tim Claeys, Guy A. E. Vandenbosch, Fellow, IEEE, Davy Pissoort, Senior Member, IEEE,
}

\begin{abstract}
In this paper, a novel algorithm that selects optimal paths for conducting automated near-field measurements is presented. The resulting dataset of measurements can then be used to model the complete near-field electromagnetic emissions of an electronic device or predict the far-field emissions. The models obtained using the training sets generated with the aid of the proposed algorithm are substantially more accurate compared to existing point-based methods. The algorithm is validated by comparing it against an earlier adaptive sampling algorithm that optimizes point-based measurement datasets.
\end{abstract}

Index Terms-Electromagnetic compatibility, near field scanning, surrogate modelling, sequential sampling

\section{INTRODUCTION}

A $\mathrm{S}$ electronic devices grow and evolve in terms of functionality and complexity, the risk of electromagnetic interference (EMI) issues increases. Therefore it becomes crucial to accurately measure/model the electromagnetic behavior of printed circuit boards (PCBs). Over the last decade, near-field (NF) scanning has emerged as an effective methodology to study the electromagnetic behavior of electronic (sub-)systems [1], [2]. NF scanning allows development of equivalent radiation models [3], [4] from the NF patterns as well as calculation of the far-field [5], [6]. Moreover, it does not require measurements to be taken in a (semi)anechoic or reverberant chamber, making it much cheaper for a manufacturer to assess the EMC behavior of their product before performing a (pre-)compliance test.

The time needed to perform a full NF scan varies according to the size of the device, but will quickly take up to several hours for a detailed data set and multiple frequencies. An automated point-based algorithm was proposed in [7]. This algorithm models the electromagnetic NF of a device using substantially less time than a regular (uniform) NF scan. The overall NF pattern is characterized by performing a minimum number of measurements and interpolating the raw NF data into a high-resolution carthography model. The algorithm selects the optimal points in the design space where measurements should be done to obtain an adequate set for the model.

P. Singh is with the Division of Scientific Computing at the Department of Information Technology, Uppsala University, Uppsala 75105, Sweden.

T. Claeys and D. Pissoort are with the Department of Electrical Engineering, ReMI Research Group, KU Leuven Technology Campus Ostend, 8400 Ostend, Belgium. (e-mail: tim.claeys@kuleuven.be)

T. Claeys and Guy A. E. Vandenbosch are with the Department of Electrical Engineering, Telecommunication and Microwaves, KU Leuven, 3000 Leuven, Belgium.

D. Pissoort is also with the Department of Electrical Engineering, Microelectronics and Sensors, KU Leuven, 3000 Leuven, Belgium.

Manuscript received April 19, 2005; revised August 26, 2015.
As an alternative, a line-based approach is considered in this work. Such an approach generates optimal lines, or paths along with measurements at pre-defined intervals to obtain a training set for the model. The paths are generated with the objective to perform space-filling exploration of the design space, and exploit non-linear regions that can be hard to model. These optimal lines not only provide better exploration and exploitation for the model than the point-based algorithm, but also lower the total path length of the complete NF measurement.

The paper is organized as follows. Section II describes the problem statement and goals. Section III briefly explains Kriging models that are a popular model type for EMC applications [8], [9], [10], and are used in this work. The proposed line-based sampling algorithm is introduced in Section IV. Sections V and VI describe the simulation setup used for the experiments, and the results, respectively. Section VII concludes the paper.

\section{GOAL STATEMENT AND PRELIMINARIES}

The goal is to generate optimal measurement paths for the probe to follow, resulting in (I) a dataset that is used to train a more accurate Kriging model than the the point-based algorithm and (II) a shorter travelling path than the pointbased algorithm. Each scan point, henceforth also called data sample, is represented as $\left(\mathbf{x}, F_{r}(\mathbf{x}), F_{i}(\mathbf{x})\right)$ where $\mathbf{x}$ is a vector that contains the horizontal, vertical; and height coordinates of the probe, and $F_{r}(\mathbf{x})$ and $F_{i}(\mathbf{x})$ represent the real and imaginary components of the electric $(E)$ or the magnetic $(H)$ field component, respectively. Each spatial coordinate in the vector $\mathbf{x}$ is denoted by a superindex $x^{(n)}$. A model is trained after obtaining data samples using a sampling algorithm. In this work Kriging models, which are shortly described in the next Section, are used.

\section{KRIGING MODELS}

Assume a set of $n$ scan points $X=\left(\mathbf{x}_{1}, \ldots, \mathbf{x}_{n}\right)^{\prime}$ in $d$ dimensions mapped to function values $\left(y_{1}, \ldots, y_{n}\right)^{\prime}$.

A Kriging model consists of two components - a regressor $h(x)$, and a centred Gaussian Process (GP) $Z$. The GP $Z$ is constructed with variance $\sigma^{2}$ and a correlation matrix $\psi$ through the residual:

$$
Y(\mathbf{x})=h(\mathbf{x})+Z(\mathbf{x}) .
$$


The $n \times p$ matrix $F$ holds the regressor $h(\mathbf{x})$ and consists of basis functions $b_{i}(\mathbf{x})$ for $i=1 \ldots p$,

$$
F=\left(\begin{array}{cccc}
b_{1}\left(\mathbf{x}_{1}\right) & b_{2}\left(\mathbf{x}_{1}\right) & \cdots & b_{p}\left(\mathbf{x}_{1}\right) \\
\vdots & \ddots & \vdots & \\
b_{1}\left(\mathbf{x}_{n}\right) & b_{2}\left(\mathbf{x}_{n}\right) & \cdots & b_{p}\left(\mathbf{x}_{n}\right)
\end{array}\right) .
$$

The $n \times n$ correlation matrix $\psi$ is defined as

$$
\psi=\left(\begin{array}{ccc}
\psi\left(\mathbf{x}_{1}, \mathbf{x}_{1}\right) & \cdots & \psi\left(\mathbf{x}_{1}, \mathbf{x}_{n}\right) \\
\vdots & \ddots & \vdots \\
\psi\left(\mathbf{x}_{n}, \mathbf{x}_{1}\right) & \cdots & \psi\left(\mathbf{x}_{n}, \mathbf{x}_{n}\right)
\end{array}\right),
$$

where $\psi\left(\mathbf{x}_{\mathbf{i}}, \mathbf{x}_{\mathbf{j}}\right)$ is a chosen correlation function, parameterized by a set of hyperparameters $\theta$. The correlation function is critical in order to train an accurate model. The Gaussian correlation function was used for experiments in this paper, which is defined as

$$
\phi\left(\theta, \mathbf{x}_{k}, \mathbf{x}\right)=\prod_{i=1}^{n} \mathrm{e}^{-\theta_{i}\left|x_{k}^{(i)}-x^{(i)}\right|^{2}},
$$

where $i$ indexes the coordinates of the probe stored in vector $\mathbf{x}$, and the parameters $\theta_{i}$ are identified by the maximum likelihood estimation. Further details can be found in [11].

\section{The Proposed LOLA-VET Algorithm}

In order to arrive at a balanced dataset that facilitates training of accurate models, the measurement locations, or paths must be carefully designed. The design space must be sufficiently explored and regions must be identified where the model might find it difficult to learn accurately. Therefore, the paths must be space-filling, and also must visit, or exploit areas of non-linearity in the design space. The information regarding non-linearity of the design space is estimated using local-linear approximations (LOLA [12]). The regions of the design space requiring space-filling information are identified using Voronoi tessellations and the sequential paths are generated by moving along Voronoi edges (Voronoi Edge Traversal - VET [13]). The exploration and exploitation components are described in detail below.

\section{A. Exploration}

To start with, the paths must be space-filling and must cover the design space evenly. Space-filling curves (Hilbert and Peano curves) [14] are ideal choices to generate initial paths for the probe to follow. Figure 1 shows a Hilbert curve of order 3. It can be seen that the curve has excellent spacefilling properties. A measuring probe can start at $(0,0)$, and measure all the way following the curve till the end at $(1,0)$.

The order of the curve can be selected based on the desired density of measurements. In practise, it is hard to estimate the order beforehand. For example, in the case of model-driven optimization, achieving good model accuracy is crucial. The number of data points required to reach a particular accuracy is different for each problem. This necessitates an iterative procedure of model training wherein additional data points are selected for training the model in each iteration. Therefore, the algorithm must be capable of iteratively generating additional

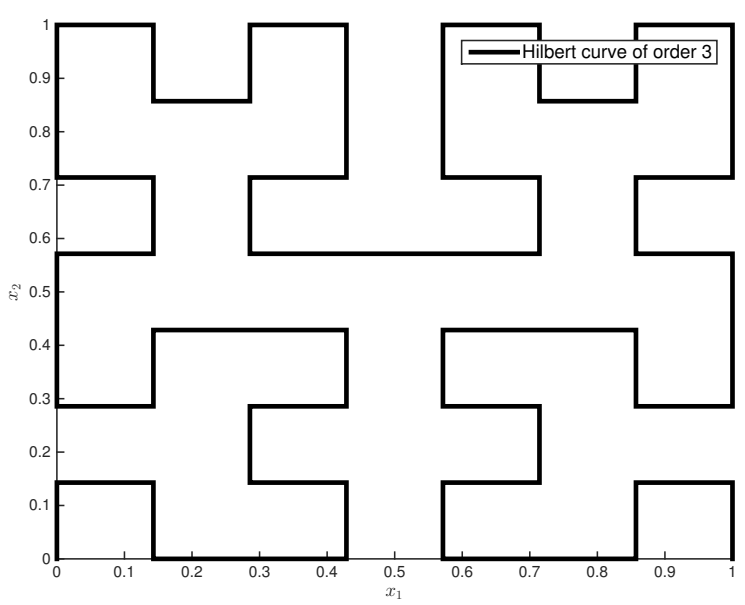

Fig. 1: A Hilbert curve of order 3.

paths that perform exploration of the design space and exploit regions of potential uncertainty.

An effective methodology to identify potential regions for exploration involves using Voronoi tessellations [12], [15]. Figure 2 shows a Voronoi tessellation of a set of data points $X=\left\{\mathbf{x}_{i}\right\}_{i=1}^{10}$. It can be observed that a Voronoi edge forms the perpendicular bisector of any two adjacent data points. Therefore, Voronoi edges form optimal paths for a probe to follow in order to gather additional measurements to supplement $X$.

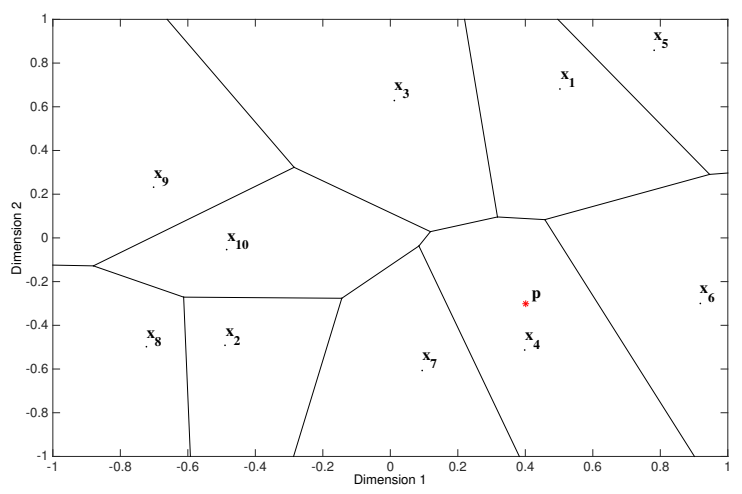

Fig. 2: A Voronoi tessellation of a set of points $X=\left\{\mathbf{x}_{i}\right\}_{i=1}^{10}$.

The probe can move from the current location (after moving along the Hilbert curve, for example) to the nearest Voronoi edge using an orthogonal projection. The probe can then follow a sequence of Voronoi edges to reach a destination point. The maximin criterion is commonly used to generate space-filling points that maximize the minimum distance from existing data points:

$$
\phi_{X}=\max _{X} \min _{\mathbf{x}_{i}, \mathbf{x}_{j} \in X}\left\|\mathbf{x}_{i}-\mathbf{x}_{j}\right\| .
$$

Maximizing the maximin or $\phi_{X}$ criterion will result in selection of points that are as far away from existing data points as possible. 
In order to uniquely identify the sequence of edges forming the path, each Voronoi edge is assiged a weight. The weight is composed of exploration and exploitation components. The exploration weight $w E_{i}$ of an edge $e_{i}$ is defined as:

$$
w E_{i}=\frac{1}{k} \sum_{j=1}^{k} \frac{1}{\operatorname{vol}\left(r_{j}\right)},
$$

where $k$ is the number of Voronoi cells sharing the edge $e_{i}$ and $\operatorname{vol}\left(r_{j}\right)$ is the (hyper)volume of the Voronoi cell $r_{j}$. he edge weights $w E_{i}$ are then normalized to the domain $[0,1]$.

\section{B. Exploitation}

The exploitation weight assigned to the edge $e_{i}$ is related to the non-linearity nearby $e_{i}$. In regions of the design space where the behaviour of the system is linear, the model can easily predict the output and fewer training data points are needed nearby. In contrast, the model will need more data points in regions where the output changes rapidly or nonlinearly.

The best indicator of linear approximation of a function $f$ is the gradient of $f$ :

$$
\nabla|f(\mathbf{x})|=\left(\frac{\delta|f(\mathbf{x})|}{\delta x^{1}}, \frac{\delta|f(\mathbf{x})|}{\delta x^{2}}, \ldots, \frac{\delta|f(\mathbf{x})|}{\delta x^{d}}\right),
$$

where $d$ is the dimensionality of the design space spanning $\left(x^{1}, x^{2}, \ldots, x^{d}\right)$. Since the gradient of the yet-to-be modeled function is not known in advance, it has to be estimated. To estimate the non-linearity associated with a Voronoi cell, a local neighborhood of $V$ points is defined around each existing data point:

$$
\begin{aligned}
& N\left(\mathbf{x}_{k}\right)=\left\{\mathbf{x}_{k v}\right\}_{v=1}^{V}, \\
& N\left(\mathbf{x}_{k}\right) \subset X \backslash\left\{\mathbf{x}_{k}\right\} .
\end{aligned}
$$

The neighbours are chosen such that they cover each direction of the design space equally well. This enables the computation of best local linear approximation $|\tilde{f}|$ at $\mathbf{x}_{k}$ :

$$
|\tilde{f}(\mathbf{x})|=\left|f\left(\mathbf{x}_{k}\right)\right|+(\nabla|f(\mathbf{x})|)_{\mathbf{x}_{k}}\left(\mathbf{x}-\mathbf{x}_{k}\right) .
$$

Assuming $A(v, \operatorname{dim})=\left(\mathbf{x}_{k v}^{\operatorname{dim}}-\mathbf{x}_{k}^{\operatorname{dim}}\right)$ and $b(v, 1)=$ $\left|f\left(\mathbf{x}_{k v}\right)\right|$ for $v=1, \ldots, V$ and $\operatorname{dim}=1, \ldots, d$, the term $(\nabla|f(\mathbf{x})|)_{\mathbf{x}_{k}}=A^{-1} b$ is computed by fitting a hyper-plane through $\mathbf{x}_{k}$, based on $N\left(\mathbf{x}_{k}\right)$.

The non-linearity in and around the Voronoi cell corresponding to data point $\mathbf{x}_{k}$ can be measured by the metric:

$$
G\left(\mathbf{x}_{k}\right)=\sum_{v=1}^{V}|| \tilde{f}\left(\mathbf{x}_{k v}\right)|-| f\left(\mathbf{x}_{k v}\right)|| .
$$

The metric $G$ is finally normalized as $\tilde{G} \in[0,1]$ :

$$
\tilde{G}\left(\mathbf{x}_{k}\right)=\frac{G\left(\mathbf{x}_{k}\right)}{G\left(\mathbf{x}_{1}\right)+G\left(\mathbf{x}_{2}\right)+\ldots+G\left(\mathbf{x}_{N}\right)},
$$

where $N$ is the total number of existing data points.

The exploitation metric associated with an edge $e_{i}$ is defined as the average normalized non-linearity score of all Voronoi cells $r_{j}$ sharing $e_{i}$, subtracted from 1 :

$$
w X_{i}=1-\frac{1}{k} \sum_{j=1}^{k} \tilde{G}\left(\mathbf{x}_{j}\right) .
$$

The subtraction is necessary since Dijkstra's shortest path algorithm [16] is used to sequentially add edges. Since Dijkstra's algorithm seeks to find a path that minimizes edge weights, a lower weight must correspond to higher exploitation. This necessitates a subtraction from 1 to invert the weights $\in[0,1]$.

\section{Combining Exploration and Exploitation}

Equations (4) and (11) are combined to obtain a metric that assigns a weight $w_{i}$ to each edge $e_{i}$ balancing exploration and exploitation:

$$
w_{i}=w E_{i}+w X_{i} .
$$

Dijkstra's shortest path algorithm [16] can now be used to generate a sequence of $n_{e}$ edges forming a path from current location to the newly generated destination point obtained using the maximin criterion. The algorithm will seek to minimize $\sum_{i=1}^{n_{e}} w_{i}$, and hence will prefer longer edges (for exploration) that traverse regions of non-linearity (for exploitation).

\section{Simulation Setup}

In order to evaluate the new algorithm, two PCBs (Figs. 3a and $3 \mathrm{~b}$ ) were simulated for 3 frequencies in an electromagnetic 3D solver [17], providing the near-fields, at a height of $4 \mathrm{~mm}$ above the PCB. The substrate (FR4) has a size of 10 by $10 \mathrm{~cm}$ and is $1.5 \mathrm{~mm}$ thick. One component, $H_{x}$, was chosen to be "scanned" by the new and old algorithm. The real and imaginary values are chosen to be modelled instead of the magnitude and phase as argumented in [10]. Evaluation of the new algorithm starts with the total scanning time. It consists of the moving time, measuring time and model building time. The moving time is calculated by multiplying the total path of the probe determined by the new an old algorithm and a typical speed of a practical scanner $(0.03 \mathrm{~m} / \mathrm{s})$. The measuring time ( $0.2 \mathrm{~s}$ per point) is multiplied by the number of measuring points. The model from the new algorithm is also evaluated on its accuracy. This is done by using the error estimates mentioned in Section VI.

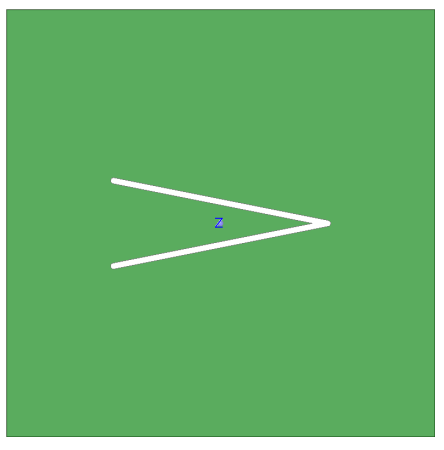

(a) PCB VLine

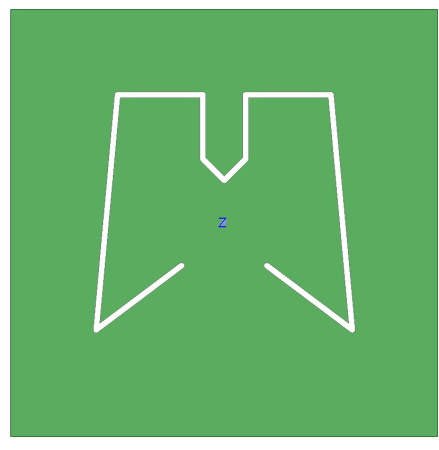

(b) PCB Random
Fig. 3: PCBs

\section{RESULTS}

For the purpose of comparison with earlier algorithms, the points-based LOLA-Voronoi algorithm was used to generate 
a set of 512 points. The near-field probe traverses the set of points in order of generation of points. The proposed LOLAVET algorithm was used to generate a path consisting of 512 points to match the earlier setup. It is interesting to note that each Voronoi vertex (as described in Sec. IV) counts as a ent point. Additionally, the near-field probe is set to perform a measurement every $10 \mathrm{~mm}$ while traversing the path generated by each algorithm.

As illustration, Kriging models trained using the LOLAVET and LOLA-Voronoi algorithms are shown in Figs. 4 and 5. The path traversed can also be seen and it can be observed that the lines added sequentially cover the space with sufficient density.

Tables I and II list the model error estimates obtained using cross-validation and a separate validation test set for the proposed algorithm, and the LOLA-Voronoi algorithm. Mean squared error (MSE), root relative squared error (RRSE), and bayesian estimation error quotient (BEEQ) [18]. BEEQ measures the improvement in error of the trained Bayesian estimator $\hat{y}$ over the prior mean $\bar{y}$, or of the updated estimate $\hat{y}$ of a recursive estimator over the predicted estimate $\hat{y}$. Mathematically, $\operatorname{BEEQ}(\hat{y})=\left(\prod_{i=1}^{n} \beta_{i}\right)^{\frac{1}{n}}$, where

$$
\beta_{i}=\frac{\left\|y_{i}-\hat{y}\left(\mathbf{x}_{i}\right)\right\|}{\left\|y_{i}-\bar{y}\left(\mathbf{x}_{i}\right)\right\|},
$$

and $y_{i}$ is the actual response value. The ideal value of BEEQ is 0 and the lower the better. The advantage of the metric BEEQ is that the effects of very large or small magnitudes on error estimates are nullified. The metrics RRSE and MSE are popular in literature and are computed as:

$$
\begin{aligned}
\operatorname{RRSE}(\hat{y}) & =\sqrt{\frac{\sum_{i=1}^{n}\left(y_{i}-\bar{y}\left(\mathbf{x}_{i}\right)\right)^{2}}{\sum_{i=1}^{n}\left(y_{i}-\hat{y}\left(\mathbf{x}_{i}\right)\right)^{2}}}, \\
\operatorname{MSE}(\hat{y}) & =\frac{\sum_{i=1}^{n}\left(y_{i}-\hat{y}\left(\mathbf{x}_{i}\right)\right)}{n} .
\end{aligned}
$$

Table III presents statistics such as probe travel distance, travel time, measurement time, modelling time, and total time taken for LOLA-VET and LOLA-Voronoi-based approaches. Modelling time includes the sampling time in addition to the model training time. It can be seen that travel distance and travel time are substantially lower for the LOLA-VET algorithm. The LOLA-Voronoi algorithm is faster in selecting samples than the LOLA-VET and this leads to faster modelling times.

For each of the two cases (real and imaginary parts of $H_{x}$ ), Tables I and II list the MSE, RRSE, and BEEQ scores corresponding to the models trained using the LOLA-VET and LOLA-Voronoi algorithms. All three scores should ideally be 0 ; therefore, lower is better. It can be inferred from the results in Tables I and II that Kriging models trained using the proposed LOLA-VET algorithm outperform the ones trained using data obtained from the LOLA-Voronoi algorithm on a separate validation set - which is a true indicator of the generalization ability of a model. For example, considering the case of the real component corresponding to the VLine microstrip at $1 \mathrm{GHz}$ frequency, the MSE score of LOLA-VET computed using the validation set is 0.000093 as opposed

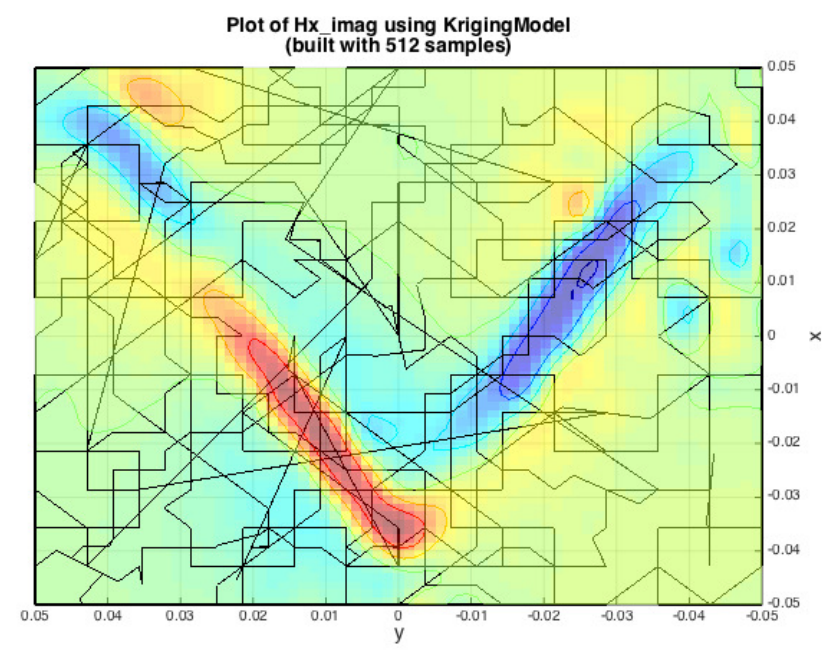

Fig. 4: The Kriging model, and the measurement paths generated using the LOLA-VET algorithm for the imaginary component of the VLine microstrip at $1 \mathrm{GHz}$ frequency and $5 \mathrm{~mm}$ measurement height.

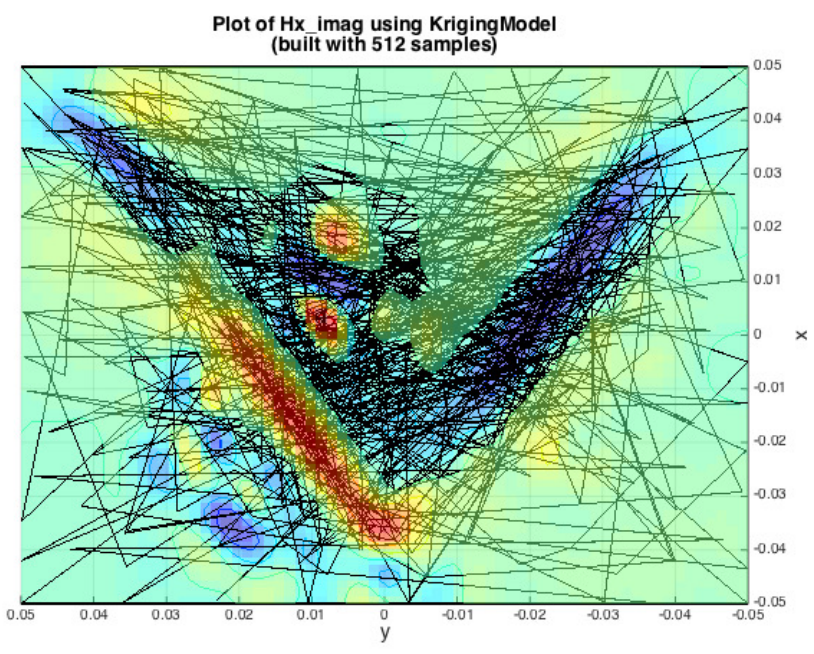

Fig. 5: The Kriging model, and the measurement paths generated using the LOLA-Voronoi algorithm for the imaginary component of the VLine microstrip at $1 \mathrm{GHz}$ freqency and $5 \mathrm{~mm}$ measurement height.

to 1.953256 corresponding to LOLA-Voronoi. There is an improvement of multiple orders of magnitude in the model obtained using LOLA-VET. Similarly, the RRSE score of 1.423883 corresponding to LOLA-VET is also more than two orders of magnitude better than 205.962772, corresponding to LOLA-Voronoi. The BEEQ score of 0.000001 corresponding to LOLA-VET, obtained using cross-validation is also an order of magnitude better than 0.000050 , corresponding to LOLAVoronoi.

It is interesting to note that cross-validation scores of LOLA-Voronoi are better than LOLA-VET in some cases. There is a substantial difference in scores corresponding to the LOLA-Voronoi algorithm between cross-validation and the validation set. In contrast, the in-sample error (cross- 
TABLE I: VLine: Model error estimates for fixed number of measurements. CV refers to cross-validation and VS refers to a validation set distinct from data used to train the models.

\begin{tabular}{|c|c|c|c|c|c|c|c|c|c|c|c|c|}
\hline \multirow[t]{3}{*}{ Test Scenario } & \multicolumn{6}{|c|}{ Real } & \multicolumn{6}{|c|}{ Imaginary } \\
\hline & \multicolumn{3}{|c|}{ LOLA-VET } & \multicolumn{3}{|c|}{ LOLA-Voronoi } & \multicolumn{3}{|c|}{ LOLA-VET } & \multicolumn{3}{|c|}{ LOLA-Voronoi } \\
\hline & MSE & RRSE & BEEQ & MSE & RRSE & BEEQ & MSE & RRSE & BEEQ & MSE & RRSE & BEEQ \\
\hline $1 \mathrm{GHz}: \mathrm{CV}$ & 0.000000 & 0.045278 & 0.000001 & 0.000005 & 0.029324 & 0.000050 & 0.000000 & 0.047452 & 0.000000 & 0.000000 & 0.035481 & 0.000039 \\
\hline $1 \mathrm{GHz}: \mathrm{VS}$ & 0.000093 & 1.423883 & - & 1.953256 & 205.962772 & - & 0.000061 & 0.891768 & - & 1.514987 & 140.854572 & - \\
\hline $5 \mathrm{GHz}: \mathrm{CV}$ & 0.000000 & 0.091909 & 0.000001 & 0.000000 & 0.040392 & 0.000066 & 0.000000 & 0.077698 & 0.000001 & 0.000000 & 0.051602 & 0.000066 \\
\hline $5 \mathrm{GHz}: \mathrm{VS}$ & 0.000676 & 5.411250 & - & 0.262579 & 106.648970 & - & 0.003225 & 6.609814 & - & 0.375374 & 71.309880 & - \\
\hline $10 \mathrm{GHz}: \mathrm{CV}$ & 0.000002 & 0.169900 & 0.000001 & 0.000000 & 0.050157 & 0.000078 & 0.000000 & 0.093468 & 0.000001 & 0.000000 & 0.042811 & 0.000059 \\
\hline $10 \mathrm{GHz}: \mathrm{VS}$ & 0.000145 & 1.805933 & - & 0.295264 & 81.506724 & - & 0.000027 & 0.960168 & - & 0.073044 & 49.990882 & - \\
\hline
\end{tabular}

TABLE II: Random: Model error estimates for fixed number of measurements. CV refers to cross-validation and VS refers to a validation set distinct from data used to train the models.

\begin{tabular}{|c|c|c|c|c|c|c|c|c|c|c|c|c|}
\hline \multirow[t]{3}{*}{ Test Scenario } & \multicolumn{6}{|c|}{ Real } & \multicolumn{6}{|c|}{ Imaginary } \\
\hline & \multicolumn{3}{|c|}{ LOLA-VET } & \multicolumn{3}{|c|}{ LOLA-Voronoi } & \multicolumn{3}{|c|}{ LOLA-VET } & \multicolumn{3}{|c|}{ LOLA-Voronoi } \\
\hline & MSE & RRSE & BEEQ & MSE & RRSE & BEEQ & MSE & RRSE & BEEQ & MSE & RRSE & BEEQ \\
\hline $1 \mathrm{GHz}: \mathrm{CV}$ & 0.000002 & 0.082148 & 0.000001 & 0.000001 & 0.033868 & 0.000054 & 0.000004 & 0.132532 & 0.000000 & 0.000003 & 0.076511 & 0.000064 \\
\hline $1 \mathrm{GHz}: \mathrm{VS}$ & 0.000049 & 0.447719 & - & 0.126396 & 22.797146 & - & 0.000056 & 0.552489 & - & 0.041983 & 15.134458 & - \\
\hline $5 \mathrm{GHz}: \mathrm{CV}$ & 0.000005 & 0.166639 & 0.000001 & 0.000001 & 0.065960 & 0.000138 & 0.000004 & 0.173212 & 0.000001 & 0.000000 & 0.141729 & 0.000098 \\
\hline $5 \mathrm{GHz}: \mathrm{VS}$ & 0.002079 & 3.730148 & $\begin{array}{c}0.00001 \\
-\end{array}$ & 0.029769 & 14.114880 & $\begin{array}{c}0.000100 \\
-\end{array}$ & 0.000482 & 1.899029 & $\begin{array}{c}0.0001 \\
-\end{array}$ & 0.134288 & 31.701443 & $\begin{array}{c}0.00000 \\
-\end{array}$ \\
\hline $10 \mathrm{GHz}: \mathrm{CV}$ & 0.000001 & 0.103441 & 0.000000 & 0.000000 & 0.049760 & 0.000192 & 0.000192 & 0.148280 & 0.000001 & 0.000000 & 0.042805 & 0.000209 \\
\hline $10 \mathrm{GHz}: \mathrm{VS}$ & 0.000422 & 1.900076 & - & 0.125790 & 32.807638 & - & 0.000825 & 2.756580 & - & 0.159989 & 38.397190 & - \\
\hline
\end{tabular}

validation score) tracks very well the generalization error (error on the validation set) in the case of the LOLA-VET algorithm, which is a true indicator of model quality. This serves to emphasize the goodness of data obtained using the LOLA-VET algorithm. Combining the results from all tables, it can be concluded that the LOLA-VET algorithm gains more information about the environment, and leads to models more accurate than those obtained by the LOLA-Voronoi algorithm while moving the probe far less. The only disadvantage of the new approach is that it is comparatively slower than LOLAVoronoi algorithm in selecting samples. For example, the time taken to perform the sampling and train the real and imaginary Kriging models for the case of the Vline microstrip (1 GHz) is $1920 \mathrm{~s}$ for LOLA-VET as compared to $1256 \mathrm{~s}$ for LOLAVoronoi. Therefore, the improvement in accuracy comes at a cost of sampling time. In comparison a standard uniform scan of this size would take up to 3600 seconds.

In summary, the proposed LOLA-VET algorithm leads to models that are substantially more accurate than the pointbased LOLA-Voronoi sampling algorithm. The LOLA-VET algorithm also gains more information per unit travelled distance as compared to the LOLA-Voronoi algorithm. The LOLA-Voronoi algorithm is faster than LOLA-VET algorithm in sampling or selecting new points.

\section{CONCLUSION}

The paper presents a novel algorithm that generates optimal paths for conducting automated measurements. The algorithm automatically balances exploration of the design space and exploitation of regions of uncertainty. The measurements obtained using a probe traversing the generated paths can be used to train a model of the electromagnetic NF of an electronic device. Experiments performed on a test problem and comparisons with an existing approach validate the proposed algorithm.

\section{REFERENCES}

[1] D. Baudry, C. Arcambal, A. Louis, B. Mazari, and P. Eudeline, "Applications of the near-field techniques in emc investigations," Electromagnetic Compatibility, IEEE Transactions on, vol. 49, no. 3, pp. 485 -493, Aug. 2007.

[2] K. Haelvoet, S. Criel, F. Dobbelaere, L. Martens, P. De Langhe, and R. De Smedt, "Near-field scanner for the accurate characterization of electromagnetic fields in the close vicinity of electronic devices and systems," in Instrumentation and Measurement Technology Conference, 1996. IMTC-96. Conference Proceedings. Quality Measurements: The Indispensable Bridge between Theory and Reality., IEEE, vol. 2, 1996, pp. 1119-1123 vol.2.

[3] D. Rinas, S. Niedzwiedz, J. Jia, and S. Frei, "Optimization methods for equivalent source identification and electromagnetic model creation based on near-field measurements," in EMC Europe 2011 York, Sept 2011, pp. 298-303.

[4] M. Johansson, H.-S. Lui, J.-C. Bolomey, and M. Persson, "Source modeling using phaseless low-frequency near-field measurements," Electromagnetic Compatibility, IEEE Transactions on, vol. 54, no. 3, pp. 613-624, June 2012.

[5] V. Volski, S. Yan, G. A. E. Vandenbosch, T. Claeys, and D. Pissoort, "Auxiliary dipoles to compensate for the finite size of the planar scanning area in near-to-far-field transformations," Electromagnetic Compatibility, IEEE Transactions on, vol. 57, no. 6, pp. 1517-1528, Dec 2015.

[6] J.-S. Lee, T.-L. Song, J.-K. Du, and J.-G. Yook, "Near-field to far-field transformation based on stratton-chu fomula for emc measurements," in Antennas and Propagation Society International Symposium (APSURSI), 2013 IEEE, July 2013, pp. 606-607.

[7] D. Deschrijver, F. Vanhee, D. Pissoort, and T. Dhaene, "Automated nearfield scanning algorithm for the emc analysis of electronic devices," Electromagnetic Compatibility, IEEE Transactions on, vol. 54, no. 3, pp. $502-510$, Jun. 2012.

[8] P. Singh, D. Deschrijver, D. Pissoort, and T. Dhaene, "Accurate hotspot localization by sampling the near-field pattern of electronic devices," IEEE Transactions on Electromagnetic Compatibility, vol. 55, no. 6, pp. 1365-1368, 2013.

[9] T. Dorné, F. Vanhee, T. Grenson, D. Pissoort, D. Deschrijver, I. Couckuyt, and T. Dhaene, "Optimized sequential sampling algorithm for emi near-field scanning," in Electromagnetic Compatibility (EMC EUROPE), 2013 International Symposium on. IEEE, 2013, pp. 385-388.

[10] T. Claeys, D. Pissoort, D. Deschrijver, I. Couckuyt, and T. Dhaene, "Sequential sampling algorithm for simultaneous near-field scanning of amplitude and phase," in 2014 International Symposium on Electromagnetic Compatibility. IEEE, 2014, pp. 79-84.

[11] I. Couckuyt, F. Declercq, T. Dhaene, H. Rogier, and L. Knockaert, "Surrogate-based infill optimization applied to electromagnetic prob- 
lems," International Journal of RF and Microwave Computer-Aided Engineering, vol. 20, no. 5, pp. 492-501, 2010.

[12] K. Crombecq, D. Gorissen, D. Deschrijver, and T. Dhaene, "A novel hybrid sequential design strategy for global surrogate modeling of computer experiments," SIAM Journal on Scientific Computing, vol. 33, no. 4, pp. 1948-1974, 2011.

[13] P. Singh, "Design of experiments for model-based optimization," Ph.D. dissertation, Ghent University, 2016.

[14] H. Sagan, Space-filling curves. Springer Science \& Business Media, 2012.

[15] P. Singh, D. Deschrijver, D. Pissoort, and T. Dhaene, "Adaptive classification algorithm for emc-compliance testing of electronic devices," Electronics Letters, vol. 49, no. 24, pp. 1526-1528, 2013.

[16] S. Skiena, "Dijkstras algorithm," Implementing Discrete Mathematics: Combinatorics and Graph Theory with Mathematica, Reading, MA: Addison-Wesley, pp. 225-227, 1990.

[17] K. Technologies, "Keysight empro software."

[18] X. R. Li and Z. Zhao, "Relative error measures for evaluation of estimation algorithms," in Information Fusion, 2005 8th International Conference on, vol. 1. IEEE, 2005, pp. 8-pp.

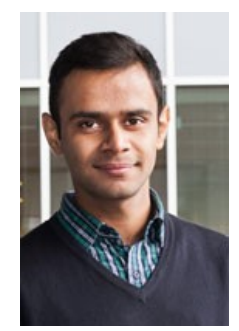

Prashant Singh received his B.Sc. (H) and M.Sc. degrees in Computer Science from the University of Delhi, India in 2009 and 2011 respectively. From July 2011 till September 2012, he worked as a Software Engineer at Nagarro Software. He received the Ph.D. degree from Ghent University in May, 2016. He is currently a post-doctoral researcher at Uppsala University, Sweden. His research interests include surrogate modeling, machine learning, scientific computing and model-based optimization.

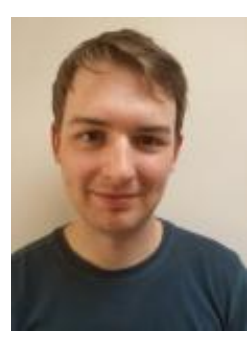

Tim Claeys was born in Tielt, Belgium, on November 8, 1990. He received the M.S. degree in industrial engineering sciences, option electronics, from the University College Katholieke Hogeschool SintLieven Gent, Ghent, Belgium, in 2013. He is currently working towards the Ph.D. degree in electrical engineering from the Katholieke Universiteit Leuven, Leuven, Belgium. Since 2013, he has been a Research Assistant at the ReMI research group (Lab FMEC), KU Leuven Technology Campus, Ostend, Belgium, which is a laboratory focusing on global reliability of electronic systems and electromagnetic compatibility. His research interests are near-field scanning, plane wave decomposition, and shielding of gaskets and other materials. 


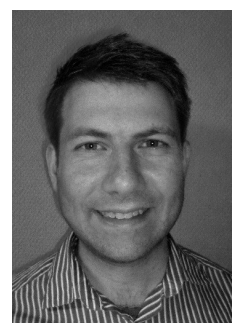

Davy Pissoort (M09, SM13) was born in 1978. $\mathrm{He}$ received theM.S. and Ph.D. degrees in electrical engineering from Ghent University, Ghent, Belgium, in 2001 and 2005, respectively. From October 2005 to October 2006, he was a Postdoctoral Researcher Ghent University, Belgium. From November 2006 to July 2009, he was a Research Engineer at the Eesof-EDA Department of Agilent Technolgies in Belgium. From August 2009 he has been the head of the research group Reliability in Mechatronics and ICT at KU Leuven, Technology Campus Ostend.

From August 2009 till Sep2016 as an Assistant Professor and since Oct 2016 as Associate Professor. His current research interests include the development of fast and efficint electromagnetic modelling methods for EMC, SI and PI, the development of characterization methods for shielding materials and gaskets, EMI Risk Management as well as the analysis and testing of the mechanical and thermal reliability of electronic modules. He is Senior Member of the IEEE EMC Society and member of the International Steering Committee of EMC Europe. In September 2013 he was vice-chair for the EMC Europe 2013 Symposium in Brugge, Belgium. In August 2015 he was co-chair for the Workshops and Tutorials at the joined IEEE EMC and EMC Europe symposium in Dresden, Germany.

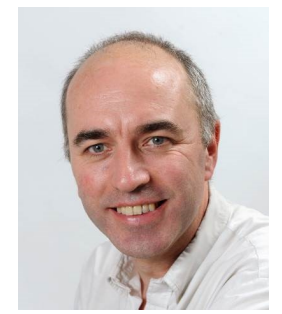

Guy A. E. Vandenbosch received the M.S. and $\mathrm{Ph} . \mathrm{D}$. degrees in Electrical Engineering from the Katholieke Universiteit Leuven, Leuven, Belgium, in 1985 and 1991, respectively. From 1991 to 1993, he held a postdoctoral research position at the Katholieke Universiteit Leuven. Since 1993, he has been a Lecturer, and since 2005, a Full Professor at the same university. Guy Vandenbosch has taught or teaches courses on ?Electromagnetic Waves?, ?Antennas?, ?Electromagnetic Compatibility?, ?Electrical Engineering, Electronics, and Electrical Energy?, and ?Digital Steer- and Measuring Techniques in Physics?. His research interests are in the area of electromagnetic theory, computational electromagnetics, planar antennas and circuits, nano-electromagnetics, EM radiation, EMC, and bio-electromagnetics. His work has been published in ca. 230 papers in international journals and has led to ca. 330 papers at international conferences. Guy Vandenbosch has been a member of the "Management Committees" of the consecutive European COST actions on antennas since 1993. Within the ACE Network of Excellence of the EU (2004-2007), he was a member of the Executive Board and coordinated the activity on the creation of a European antenna software platform. At present, he leads the EuRAAP Working Group on Software and represents this group within the EuRAAP Delegate Assembly. From 2001 to 2007, he was the President of SITEL, the Belgian Society of Engineers in Telecommunication and Electronics. From 2008 to 2014, he was a member of the board of FITCE Belgium, the Belgian branch of the Federation of Telecommunications Engineers of the European Union. In the period 1999-2004, he was vice-chairman, and in the period 20052009 secretary of the IEEE Benelux Chapter on Antennas en Propagation. Currently he holds the position of chairman of this Chapter. In the period 2002-2004 he was secretary of the IEEE Benelux Chapter on EMC. In the period 2012-2014, he was secretary of the Belgian National Committee for Radio-electricity (URSI), where he is also in charge of commission E. Guy Vandenbosch is a fellow of the IEEE. From September to December 2014, he was a visiting professor at Tsinghua University, Beijing, China. 